\title{
INTERNET Y SU IMPACTO EN LA ENSEÑANZA - APRENDIZAJE
}

\author{
INTERNET AND ITS IMPACT ON TEACHING - LEARNING
}

\section{MAURICIO GARZÓN RODAS', DANNY FIGUEROA CEDEÑO²}

\author{
$1 \quad$ Facultad de Especialidades Empresariales, Universidad Católica de Santiago de Guayaquil \\ mauricio.garzon@cu.ucsg.edu.ec \\ 2 Facultad de Especialidades Emrpesariales, Universidad Católica de Santiago de Guayaquil \\ danny.figueroa01@cu.ucsg.edu.ec
}

RESUMEN

Esta investigación realizó una prueba piloto con diez estudiantes, de una población total de 94, que tomaron la materia Informática I de la carrera de Comercio y Finanzas Internacionales Bilingüe de la facultad de Especialidades Empresariales de la Universidad Católica de Santiago de Guayaquil en el semestre A-2014, con el objetivo de implementar un sistema de educación en línea para el aprendizaje de esta materia. Sus objetivos específicos fueron incluir el concepto de la educación en línea y los criterios para la elección de esta modalidad, ofrecer normas para la organización del sistema en línea, aportar contenidos para la producción de materiales y para la organización tutorial, diseñar actividades interactivas que atraigan una variedad de estilos de aprendizaje y diseñar la propuesta metodológica para la modalidad en línea de la materia Informática I. Se utilizó la plataforma Moodle de la Universidad Católica de Santiago de Guayaquil, y la herramienta de Microsoft Excel para la tabulación de las encuestas y gráficos estadísticos. La propuesta metodológica para el aprendizaje desde la web, planteada en este proyecto de investigación fue viable, no sólo por el éxito que se tuvo en la prueba piloto realizada con los diez estudiantes, donde aprobaron todos, sino también por la información obtenida a través de las encuestas realizadas, que sustenta que la educación en línea es actualmente una de las metodologías más utilizadas para ofrecer cursos de manera exitosa.

PALABRAS CLAVE: educación, enseñanza, aprendizaje, internet, web.

\section{ABSTRACT}

This research conducted a pilot study with 10 students, out of a population of 94 , taking the subject Informatica / as a regular course of the career of Comercio y Finanzas Internacionales Bilingue at Universidad Catolica de Santiago de Guayaquil, in semester A-2014, in order to implement a system of online education for learning in this subject. Its specific objectives were to include the concept of online education and the criteria for the choice of this modality, to provide guidelines for the organization of the online courses, to provide content for the production of materials and for tutorial activities, to design interactive activities that appeal a variety of learning styles and to design a methodology for the online course of the subject Informatica I. The Moodle platform of the Universidad Catolica de Santiago de Guayaquil, and Microsoft Excel tool for tabulating surveys and charts were used. The proposed learning methodology for this subject in this research project was feasible, not only for the success they had in the pilot study with 10 students, where all of them approved, also by the information obtained through surveys, which holds that online education is currently one of the methodologies more used to provide online courses successfully.

KEYWORDS: education, teaching, learning, internet, web. 
INTRODUCCIÓN

Desde la creación de la carrera de Comercio y Finanzas Internacionales Bilingüe se ha acostumbrado a que las materias curriculares y $\mathrm{co}^{-}$ curriculares sean parte de nuestra vida clásica, ya sea como docente o estudiante. Se denomina clásica por el hecho de que implica salir de la casa e ir a sentarse a una sala de informática a escuchar a un docente que imparte clases, cumpliendo con un horario establecido.

Actualmente, la comunidad estudiantil en la UCSG crece a pasos agigantados, lo que hace que la infraestructura cada vez se haga menos eficiente y en muchos casos afecte a la distribución de los paralelos. Mientras más estudiantes ingresen, mayor va a ser el número de paralelos que se tengan que abrir, mientras que se cuenta con la misma cantidad de aulas informáticas, con esto puede ocurrir: que no se abran todos los paralelos que debieran, que se abran los paralelos y que se paralicen las clases hasta encontrar disponibilidad de aulas en otra unidad académica, que las aulas pasen copadas con cursos, o que los estudiantes no tengan donde hacer tareas o investigaciones, entre otros.

La Universidad Católica de Santiago de Guayaquil se ha caracterizado por ser un centro de educación superior que se ha preocupado por mantenerse a la vanguardia tecnológica y en constante actualización, sobre todo en esta era digital, un ejemplo de esto es que desde hace varios años la Universidad cuenta con una plataforma informática web que integra procesos académicos y administrativos, lo que le ha permitido automatizar procesos y compartir tareas con la comunidad universitaria, como por ejemplo, el ingreso de asistencias y notas, antes realizado por personal administrativo, y ahora por los docentes a través de un portal web; o como las inscripciones de estudiantes, antes realizadas de manera presencial en la universidad, ahora desde cualquier sitio a través de internet. Otro ejemplo de vanguardia tecnológica es que la Universidad mantiene dotadas sus diferentes facultades con laboratorios de informática y salas de profesores que tienen equipos de última generación y acceso a Internet de alta velocidad, facilitando así la investigación de docentes y alumnos.

Sin duda alguna las Tecnologías de Información y Comunicación (TIC) han impactado de manera significativa en los procesos académicos/administrativos de la Universidad, han facilitado la investigación por de docentes y alumnos mediante el acceso a internet de alta velocidad y han modificado las tradicionales metodologías de enseñanza y aprendizaje al disponer de recursos audiovisuales y nuevos medios de enseñanza para el dictado de clases y el envío de trabajos.

\section{LAS TICS Y LOS CAMBIOS EN LA EDUCACIÓN SUPERIOR}

Los cambios que se dan en las instituciones de educación superior presentan tres manifestaciones que podemos considerar como respuestas de gran interés desde la práctica, para comprender el fenómeno, todas ellas interrelacionadas dentro de los procesos de innovación: cambios en el rol del profesor, cambios en el rol del alumno y cambios en el proceso de enseñanza-aprendizaje (cambios metodológicos) (Salinas, 2007).

CAMBIOS EN EL ROL DEL PROFESOR El rol del profesor cambia de la transmisión del conocimiento a los alumnos a ser facilitador en la construcción del propio conocimiento por parte de estos. El profesor deja de ser fuente de todo conocimiento y pasa a actuar como guía de los alumnos para facilitarles el uso de recursos y herramientas que necesitan para explorar y elaborar nuevo conocimiento y destrezas, pasa a actuar como gestor de los recursos de aprendizaje y acentuar su papel de orientador.

\section{CAMBIOS EN EL ROL DEL ALUMNO}

El enfoque tradicional ha consistido en acumular la mayor cantidad de conocimientos posible, pero en un mundo rápidamente cambiante esto no es eficiente, al no saber si lo que se está aprendiendo será relevante.

El alumno ya se encuentra en el contexto de la sociedad de la información y no se adapta al papel que tradicionalmente se le ha adjudicado, es indudable que el contacto con las TIC lo beneficia de muchas maneras, sin embargo se requiere de acciones educativas relacionadas con el uso, selección, utilización y organización de la información de forma que pueda irse formando como un ciudadano maduro de la sociedad de la información. El apoyo y la orientación que recibirá, así como la diferente disponibilidad tecnológica, son elementos cruciales en la explotación de las TIC para actividades de formación.

CAMBIOS METODOLÓGICOS

Frecuentemente se ha procurado reproducir los modelos de enseñanza-aprendizaje dominantes encontrándose muchos cursos y experiencias 
que se basan fundamentalmente en el modelo clásico de enseñanza-aprendizaje. Las posibilidades de las TIC permiten reproducir de alguna forma estos modelos y en algunos casos puede entenderse que ésta sea la opción 'adecuada' (la adecuada combinación de elementos tecnológicos, pedagógicos y organizativos). No se inventan nuevas tecnologías, sino que la utilización de las TIC en educación abre nuevas perspectivas respecto a una enseñanza mejor, apoyada en entornos en línea, cuyas estrategias son prácticas habituales en la enseñanza presencial, pero que ahora son simplemente adaptadas y redescubiertas en su formato virtual. (Salinas J. ,2004).

\section{DEFINICIONES DE E-LEARNING}

Para Vega (2007), e-Learning es un proceso de enseñanza-aprendizaje mediado a través de las TICs, formado por un conjunto de metodologías pedagógicas y de comunicación, gestión de contenidos formativo y organización educativa.

A continuación se menciona cuatro definiciones de esta modalidad de educación (Mendoza, 2003):

1. E-Learning es el suministro de programas educacionales y sistemas de aprendizaje a través demedios electrónicos. E-Learning se basa en el uso de una computadora $\mathrm{u}$ otro dispositivo electrónico (por ejemplo, un teléfono móvil) para proveer a las personas de material educativo. La educación a distancia creó las bases para su desarrollo, el cual viene a resolver algunas dificultades en cuanto a tiempos, sincronización de agendas, asistencia y viajes, problemas típicos de la educación tradicional.

2. Técnicamente, el $e$-Learning es la entrega de material educativo vía medios electrónicos, incluyendo el internet, intranet, extranet, audio, vídeo, red satelital, televisión interactiva, CD y DVD, entre otros medios.

3. Para los educadores, $e$-Learning es el uso de tecnologías de redes y comunicaciones para diseñar, seleccionar, administrar, entregar y extender la educación.

4. De forma descriptiva, la educación electrónica es la capacitación y adiestramiento de estudiantes y empleados usando materiales disponibles para Web a través del Internet, llegando a ofrecer sofisticadas facilidades como flujo de audio y vídeo, presentaciones en PowerPoint, vínculos a información relativa al tema publicada en la web, animación, libros electrónicos y aplicaciones para la generación y edición de imágenes.

Vega (2007) señala que en función de las características y necesidades del proyecto formativo dentro de cada institución, cualquier programa de $e$-Learning está conformado por la previa definición de:

a. El modelo organizacional: la implantación de procesos de $e$-Learning implica modificaciones en la organización educativa, por lo que se sugiere la realización de los siguientes pasos:

- Diagnóstico: detectar las necesidades institucionales que se desean superar con la aplicación de proyectos de $e$-Learning.

- Comunicación interna: es muy importante crear una política interna de comunicación, en que se involucre a los diferentes miembros de la institución, para dar a conocer los cambios organizativos que se producen al generar proyectos de $e$-Learning, con la intención de evitar, en la medida de lo posible, las actitudes de resistencia al cambio.

- Desarrollo del proyecto de e-Learning: con los datos obtenidos, producto del diagnóstico, el grupo de trabajo tomará las decisiones con respecto a las estrategias educativas, programa académico, diseño de contenidos y materiales complementarios de la acción formativa.

- Diseño de la interfaz: definir el tipo de estructura y los medios de interacción.

- Seguimiento y control del proyecto: revisar la calidad educativa de la acción formativa, garantizar la formación permanente de los formadores, supervisar las buenas prácticas de los docentes, evaluar el desempeño académico del estudiante y proporcionar apoyo técnico permanentemente.

b. El modelo pedagógico: aunque no existe una taxonomía única para definir un modelo pedagógico institucional de $e$-Learning, sí existen elementos comunes que hay que seguir a la hora de delimitarlo:

- Conocimiento de las expectativas del aprendizaje: al inicio de la acción formativa se dan a conocer a los estudiantes el tipo de conocimientos que se espera obtener de la misma. 
- Estrategias de enseñanza-aprendizaje: se proponen estrategias individuales y colectivas, que propicien la búsqueda de información, la interacción, la retroalimentación y el aprendizaje colaborativo a través de las TIC.

- Claridad y exactitud en la delimitación de los objetivos de aprendizaje: en ellos se definirán los conocimientos, habilidades y competencias que alcanzarán los estudiantes.

- Contenidos: los contenidos deben aparecer ordenados, han de ser relevantes, pertinentes y estar actualizados en relación a los objetivos de aprendizaje previamente definidos.

- Actividades de aprendizaje: delimitación de actividades que contribuyan al cumplimiento de los objetivos de aprendizaje, a través del uso de las TIC.

- Evaluación del aprendizaje: en este punto hay que considerar las más recientes propuestas en torno al tema de la evaluación del aprendizaje y tener en cuenta la forma de seleccionar y organizar los instrumentos de evaluación. De igual forma, hay que considerar los aspectos relacionados con la tecnología a través de la cual se va a evaluar.

- Tutoría: definir el tipo de dinámicas de atención, interacción y retroalimentación que se le va a otorgar al estudiante sobre motivación al estudio, contenido y metodología de trabajo.

c. El modelo tecnológico: en función del perfil de los formadores, profesores y los estudiantes, las características de los contenidos y el tipo de objetivos académicos a alcanzar, se podrá seleccionar las TICs que mejor se ajusten a las necesidades institucionales.

- Interacción: selección de la(s) tecnología(s) y planificación de la dinámica de interacción.

- Administración: definir el proceso administrativo automatizado adecuado para generar un sistema de control y seguimiento de los usuarios.

- Privacidad: crear un sistema que garantice la seguridad, privacidad e integridad de la información.

- Acceso: diseñar unos estándares de acceso de acuerdo con las características de los contenidos y actividades e-learning. Faci- litar el acceso a través de una navegación intuitiva y sencilla para los usuarios, y reducir, dentro de lo posible, el tiempo de descarga de la información.

- Orientación: disponer de elementos que ayuden al usuario en todo momento a ubicarse en la navegación.

- Orden: ofrecer una organización lógica de los contenidos.

A continuación se presentan los resultados que se obtendrán si se toma la materia Informática I en la modalidad online:

- La materia se puede ver desde el lugar de residencia, algún cyber, o en alguna computadora de las salas de informática de la facultad. No se necesita trasladarse a lugares distantes o fuera del alcance del estudiante. Con la nueva propuesta del programa de Informática I en línea, es posible obtener la aprobación de la materia sin tener que cumplir con un horario establecido y presencial en los predios universitarios.

- El programa educativo en línea permite tomar el curso en el tiempo disponible y a un ritmo conveniente.

- El contenido de los programas al estar en línea se pueden actualizar continuamente, esto hace que la información que se presenta sea actualizada y relevante.

- Se disminuye notablemente el uso de las salas de cómputo para clases, dejando así disponibilidad para trabajos e investigaciones.

- Se puede atender la programación a paso individualizado, lo que hace que la retención del conocimiento sea alta, el programa educativo contará con registro de actividades que permitirán que se consideren otros puntos de vista y se permitan revisarlas tantas veces como sea necesario.

- Las formas de contacto con los facilitadores o profesores son muchas: chat, foros de discusión, mensajes privados, consulta en vivo vía conferencia online, etc.

- El estar frente a una computadora permitirá acceder inmediatamente a otras fuentes de consulta para complementar las lecturas, apoyarse de sitios como Google académico y bibliotecas virtuales, entre otros. de forma inmediata en el mismo momento que se está aprendiendo. 
MATERIALES Y MÉTODOS

Se utilizó la siguiente metodología: para llevar a cabo el análisis estadístico, se tomó una muestra que representa el criterio más acercado a la realidad sobre la opinión de los estudiantes que realizaron el curso de Admisión de Invierno 2014 y que se inscribieron en el semestre A2014 en la materia de Informática I en la carrera de Comercio Finanzas Internacionales Bilingüe. La población fue de 125 estudiantes, información que se obtuvo de la secretaría de la carrera.

Se utilizó la fórmula para poblaciones finitas y nos dio 94 estudiantes a encuestar y que representó a la totalidad de la población. Esto se lo realizó para las encuestas que se aplicó a los estudiantes al inicio, antes que se realiza la prueba piloto.

También se realizó otro tipo de encuestas a cuatro profesores, dos de ellos coordinadores del Sistema de Educación a Distancia y dos profesores del área de informática de la carrera de Comercio y Finanzas Internacionales Bilingüe.

Adicionalmente se realizó una encuesta de satisfacción a todos los estudiantes que participaron en la prueba piloto del curso de Informática I en línea. Esto fue al finalizar el mismo donde intervinieron 10 estudiantes.

Para este proyecto se utilizó la plataforma Moodle que utiliza la UCSC para las tutorías virtuales, tanto presenciales como no presenciales.

Se utilizó la herramienta de Excel para la tabulación de las encuestas y gráficos estadísticos.

Los métodos y técnicas de investigación fueron cuantitativos, debido a que la recogida de información se la realizó mediante encuestas antes y después del curso piloto de Informática I en línea.

RESULTADOS

Elegimos la educación electrónica (e-Learning) para la ejecución de la prueba piloto para que los estudiantes puedan ver la materia de Informática I en línea. La herramienta que se utilizó para la implementación fue Moodle, ya que es la que utiliza la UCSG para sus tutorías virtuales en clases presenciales y para clases a distancia. Esto fue corroborado en las encuestas realizadas a los profesores que indicaron que de preferencia sea la herramienta que utiliza la UCSG, tal como se ve en la Tabla 1.
TABLA 1. PROFESORES QUE CREEN QUE LA PLATAFORMA MOODLE ES ADECUADA PARA IMPARTIR LA MATERIA INFORMÁTICA I EN LÍNEA.

\begin{tabular}{ll}
\hline SÍ & 4 \\
NO & 0 \\
NO CONTESTARON & 0 \\
\hline
\end{tabular}

Adicionalmente, de los resultados de las encuestas a estudiantes y profesores, se obtuvo, que los docentes utilizaban las diferentes opciones que ofrece la herramienta Moodle para interactuar con sus estudiantes. Lo que implica que los estudiantes ya conocían cómo manejar la herramienta Moodle y utilizaban las mismas opciones para poder revisar, trabajar y enviar sus tareas solicitadas por su profesor, tal como muestran la Tablas 2 y 3 de las encuestas que se realizaron a estudiantes y profesores respectivamente.

TABLA 2. ESTUDIANTES QUE HAN RECIBIDO Y CONTESTADO TAREAS POR MEDIO DE LA PLATAFORMA DE LA TUTORÍA VIRTUAL.

\begin{tabular}{lr}
\hline SÍ & 92 \\
NO & 1 \\
NO CONTESTARON & 1 \\
\hline
\end{tabular}

TABLA 3. OTRAS OPCIONES DENTRO DE LA HERRAMIENTA MOODLE QUE UTILIZARÍAN LOS PROFESORES COMO APOYO PARA LAS CLASES EN LÍNEA.

\begin{tabular}{lc}
\hline FOROS & 0 \\
TAREAS & 0 \\
CUESTIONARIOS & 0 \\
ENCUESTAS & 0 \\
TODAS LAS MENCIONADAS & 4 \\
NO CONTESTARON & 0 \\
\hline
\end{tabular}

Los estudiantes 94 encuestados de la muestra seleccionada, 92 respondieron afirmativamente que sí han utilizado la tutoría virtual. Solo dos estudiantes no lo han hecho. Por lo tanto decimos que los estudiantes conocen la plataforma que utiliza la Universidad y le pueden sacar provecho para ver la materia en forma virtual. Esto se muestra en la siguiente Tabla 4.

TABLA 4. ESTUDIANTES QUE HAN UTILIZADO LA PLATAFORMA MOODLE (TUTORÍA VIRTUAL) PARA ALGUNA DE SUS CLASES.

\begin{tabular}{lr}
\hline SÍ & 94 \\
NO & 2 \\
NO CONTESTARON & 1 \\
\hline
\end{tabular}


De los 94 estudiantes encuestados de la muestra seleccionada, la mayoría dicen que la materia de Informática I sí se la puede ver en línea. Esto indica que sí hay una aceptación por parte de ellos. De la misma manera así lo indican los profesores encuestados, tal como se muestra en las Tablas 1 y 5.

TABLA 5. ESTUDIANTES QUE CREEN QUE SE PUEDE IMPARTIR LA MATERIA INFORMÁTICA I EN LÍNEA.

\begin{tabular}{lc}
\hline SÍ & 78 \\
NO & 16 \\
\hline NO CONTESTARON & 0 \\
\hline
\end{tabular}

Tal como se ve en la Tabla 6, la mayoría de los estudiantes indican que desearían ver la materia de Informática I en línea con videos de cada clase, lo que a nuestro criterio es lo más apropiado. Así también lo indican los profesores, tal como se muestra en la Tabla 7.

TABLA 6. METODOLOGía QUE DESEARÍAN LOS ESTUDIANTES PARA LA MATERIA INFORMÁTICA I EN LÍNEA.

\begin{tabular}{lc}
\hline VIDEOS & 53 \\
MANUALES & 20 \\
TEMAS DE CLASE & 5 \\
NO CONTESTARON & 16 \\
\hline
\end{tabular}

TABLA 7. METOdología QUE UtILIZARÍA LOS DOCENTES PARA IMPARTIR LA MATERIA INFORMÁTICA I EN LÍNEA.

\begin{tabular}{lc}
\hline VIDEOS & 4 \\
MANUALES & 0 \\
TEMAS DE CLASE & 0 \\
\hline NO CONTESTARON & 0 \\
\hline
\end{tabular}

A continuación se muestra en la Figura 1, la pantalla de ingreso a la plataforma que se utilizó para el curso de Informática I en línea.
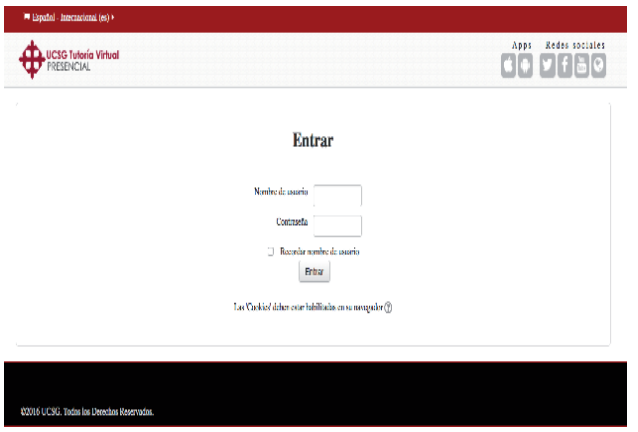

Figura 1. UCSG Tutoría Virtual
En todas las semanas se ingresaron a la plataforma los videos explicativos, que fueron obtenidos desde la web, de cada clase a partir de la semana del 26 de mayo al 28 de septiembre, en la cual se explica claramente las opciones de Word y Excel que corresponden al programa de estudio de la materia de Informática I.

Se ingresaron al curso de Informática I en línea 78 videos, de los cuales 8 videos son temas que explican herramientas de Word y 70 videos son temas que explican herramientas de Excel. Estos videos fueron obtenidos desde el Internet $y$ se especifican al final en las referencias bibliográficas. También se ingresaron diez tareas interactivas que se crearon, de los cuales cuatro fueron tipo cuestionario y 6 fueron de ejercicios aplicados para lo cual se tuvo que ingresar seis archivos que explicaban como realizar cada tarea. Adicionalmente se ingresó dos archivos para que se realice los exámenes, dos tareas para la realización de los exámenes y una tarea para la encuesta de satisfacción del curso.

A través de la Tutoría Virtual se envió a los estudiantes diez tareas interactivas, las cuales fueron realizadas por ellos y obtuvieron buenas notas, tal como se muestra en la Tabla 8 .

TABLA 8. NOTAS DE LOS ESTUDIANTES DEL CURSO (PRUEBA PILOTO) DE INFORMÁTICA I EN LÍNEA.

\begin{tabular}{lrrrr} 
ESTUDIANTES & $\begin{array}{r}\text { 1ER. } \\
\text { PARCIAL }\end{array}$ & $\begin{array}{r}\text { 200. } \\
\text { PARCIAL }\end{array}$ & MEJORAMIENTO & $\begin{array}{r}\text { PROMEDIO } \\
\text { FINAL }\end{array}$ \\
\hline Estudiante 1 & 8.75 & 7.75 & & 8.25 \\
\hline Estudiante 2 & 7.75 & 6.50 & & 7.13 \\
\hline Estudiante 3 & 9.00 & 5.75 & & 7.38 \\
\hline Estudiante 4 & 10.00 & 8.50 & & 9.25 \\
\hline Estudiante 5 & 9.50 & 9.75 & & 9.63 \\
\hline Estudiante 6 & 9.00 & 9.00 & & 9 \\
\hline Estudiante 7 & 9.50 & 8.50 & 10.00 & 9.75 \\
\hline Estudiante 8 & 9.75 & 7.00 & 7.00 & 8.38 \\
\hline Estudiante 9 & 8.50 & 6.75 & & 7.63 \\
\hline Estudiante 10 & 8.00 & 8.5 & & 8.25 \\
\hline
\end{tabular}

\section{DISCUSIÓN}

Basado en los datos obtenidos podemos decir que el curso de Informática I en línea fue exitoso, ya que de los estudiantes que participaron todos aprobaron el curso. El contenido de la materia Informática I fue ingresado a la Tutoría Virtual de la UCSG, en el cual se explica todos los temas que incluye el curso, paso a paso. 
Una vez que culminó el curso de Informática I en línea, quedaron satisfechos por haber aprobado la materia y haberla visto, utilizando esta nueva metodología. Incluso afirmaron que recomendarían a sus compañeros, si posteriormente hay la opción, para que tomen la materia en línea, tal como se ve en la Tabla 9.

tABLA 9. ESTUDIANTES QUE RECOMENDARÍAN CURSAR LA MATERIA DE INFORMÁTICA I EN LÍNEA.

\begin{tabular}{lr}
\hline SÍ & 10 \\
NO & 0 \\
NO CONTESTARON & 0 \\
\hline
\end{tabular}

Vemos entonces que según los resultados obtenidos, esta metodología de enseñanza - aprendizaje en línea es totalmente viable para ser aplicada y tenerla como una opción más para los estudiantes.

\section{CONCLUSIONES}

El impacto que tiene el Internet en la enseñanza es positivo, ya que como vimos la educación en línea es un medio que actualmente es utilizado para la enseñanza aprendizaje de diferentes temas, y es por esto que se realizó un estudio donde se realizó una prueba piloto de un curso de Informática I en línea para la carrera de Comercio y Finanzas Internacionales Bilingüe de la UCSG, de la cual se sacó las siguientes conclusiones:

- Por ser Informática I una materia co-curricular que se imparte en todas las carreras de la Universidad; así como que los estudiantes en su mayoría ya conocen las herramientas de Word y Excel es una buena opción que exista la posibilidad que se incluya esta materia para que los estudiantes, que deseen, la puedan ver en línea.

- La prueba piloto realizada a los diez estudiantes que se inscribieron en la materia Informática I paralelo A del semestre A2014 de la carrera de Comercio y Finanzas Internacionales Bilingüe, nos indica que en base a los resultados obtenidos en cuanto a las calificaciones y las opiniones de los estudiantes y profesores, sería una buena opción que esta materia pueda ser tomada en línea.

- La inclusión de videos explicativos de temas relacionados a Word y Excel utilizada en esta prueba dio resultados en proceso de enseñanza aprendizaje, así como el envío de tareas interactivas para que sean realizadas por los estudiantes.
Entonces podemos decir que la metodología aplicada para la educación en línea no es algo nuevo y es una alternativa que tenemos al momento de realizar un curso. En la actualidad hay muchos cursos en línea que se ofrecen por la Web o por anuncios de diferentes medios que generalmente está dirigida a personas que por distintas razones no cuentan con el tiempo necesario para realizar un curso de manera presencial. Por lo tanto concluimos que, para el estudio realizado, la materia de Informática I puede ser dada en línea para los estudiantes que así lo requieran.

\section{REFERENCIAS BIBLIOGRÁFICAS}

Barker, A., Krull, G., and Mallinson, B (2005). A proposed theoretical model for m-learning adoption in developing countries. Consultado el 1 de septiembre del 2014. Obtenido de: http:// www.mlearn.org.za/CD/papers/Barker.pdf

Bates, P. (2003). T-learning. A study into TV-based interactive learning to the home. Consultado el 5 de septiembre del 2014. Obtenido de: ftp:// ftp.cordis.europa.eu/pub/ist/docs/ka3/eat/tlearning.pdf

Cruz R., López G. (2007). Una visión general del m-learning y su proceso de adopción en el esquema educativo. Universidad Autónoma de Baja California México. Consultado el 5 de septiembre del 2014. Obtenido de: http://cux. uaemex.mx/ renecruz/papers/Paper2-CruzFlores.pdf

Isla J., Ortega, F. (2014); Las nuevas tecnologías en la comunicación humana: La videoconferencia en la educación. Consultado el 1 de septiembre del 2014. Obtenido de: http://www. corten.org/portal/index.php/33-articulos/ redes-y-comunidades-virtuales/136-las-nuevas-tecnologias-en-la-comunicacion-humanala-videoconferencia-en-la-educacion

González G., Chacón X. (2011). Control de calidad en los cursos en línea de un programa en Ingeniería Informática. Universidad Estatal a Distancia (UNED). Consultado el 12 de septiembre del 2014. Obtenido de: http://www.udgvirtual. udg.mx/apertura/index.php/apertura/article/ view/213/228

Llorente M. (2006). El tutor en E-learning: aspectos a tener en cuenta. Edutec. Revista Electrónica de Tecnología Educativa. Consultado el 12 de septiembre del 2014. Obtenido de: http://edutec.rediris.es/Revelec2/revelec20/llorente.htm 
Mendoza, J. (2003). Concepto de E-learning| Educación a distancia. Consultado el 12 de septiembre del 2014. Obtenido de http://www.informaticamilenium.com.mx/es/articulos/soluciones/82concepto-de-e-learning-educacion-a-distancia. html

Rubio A. (2003). Internet y Enseñanza: La Educación Virtual. Edipo,S.A. Consultado el 12 de septiembre del 2014. Obtenido de: http:// pendientedemigracion.ucm.es/info/hcs/angel/ articulos/internetyensenanza.pdf

Salinas, J. (2004). Integración de las TIC en las instituciones de educación superior como proyectos de innovación educativa. Consultado el 12 de septiembre del 2014. Obtenido de: http:// eva.universidad.edu.uy/pluginfile.php/297073/ mod_resource/content/1/PARA\%20SUBIR.pdf
Santamarina R. (2003). Reflexiones sobre educación a distancia. Petrotecnia "El Conocimiento". Consultado el 15 de septiembre del 2014. Obtenido de: http://biblioteca.iapg.org.ar/ArchivosAdjuntos/Petrotecnia/2003-1/Educacion. pdf

Sariola J., Sampson J., Vuorinen R., and Kynaslahti H. (2001). Promoting mlearning by the uniwap Project within higher education. Consultado el 15 de septiembre del 2014. Obtenido de: http:// www.icte.org/T01_Library/T01_261.pdf

Vega, R. (2007), 'Estado del arte del E-learning', en Buenas Prácticas de E-learning, Ed Landeta, A., ANCED: Asociación nacional de centros de e-learning y distancia, Madrid. 